\title{
Planificación familiar y fecundidad de la población indígena en el México urbano
}

\section{Family planning and indigenous population fertility in urban Mexico}

\author{
Germán Vázquez-Sandrin y Elsa Ortíz-Ávila \\ Universidad Autónoma del Estado de Hidalgo, México
}

\section{Resumen}

En el artículo se busca analizar la relación entre los niveles de fecundidad de tres generaciones de población indígena, el tipo de parto, el lugar del parto y el uso de métodos anticonceptivos para describir cómo ha influido en estas generaciones la medicalización de la fecundidad. Asimismo, se explora la secuencia de los eventos anteriormente mencionados en el curso de vida de las mujeres indígenas y se ajusta un modelo logístico con tiempo discreto. La fuente de información utilizada es la Encuesta Demográfica Retrospectiva de 2011. Los resultados muestran que las trayectorias asociadas con la medicalización de la fecundidad, en especial la atención del parto en un hospital y el parto por cesárea, están fuertemente asociadas con la disminución de la descendencia tanto en las mujeres indígenas como en el total de mujeres.

Palabras clave: Indígenas, fecundidad, anticoncepción, México, demografía.

\section{Abstract}

This article aims to analyze the relationship between the three indigenous groups of generations fertility levels, the type of birth, the place of birth and the use of contraceptive methods to describe how it has influenced these generations the fertility medicalization. In the same way, the events sequence mentioned above in the life course of indigenous women is explored and a logistic model is adjusted. The data source used is the Retrospective Demographic Survey of 2011. The results showed that the trajectories associated with the fertility medicalization, especially hospital birth and cesarean birth, are strongly associated with the offspring reduction in both indigenous and total women.

Keywords: Indigenous people, fertility, contraception, Mexico, demography. 


\section{INTRODUCCIÓN}

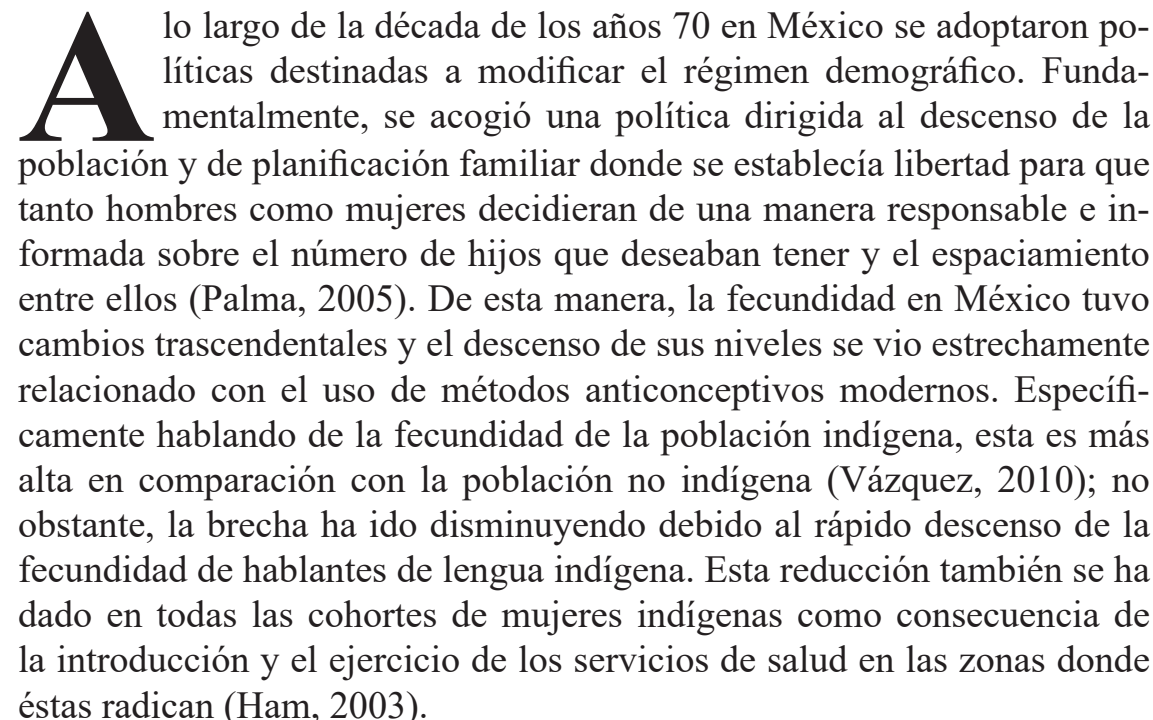

Es así que, el presente artículo pretende estudiar la relación entre los niveles de la fecundidad por cohorte de nacimiento de la población indígena, el tipo de parto, el lugar del parto, el uso de métodos anticonceptivos y el efecto de variables asociadas al nacimiento anterior en la disminución de la descendencia para describir cómo ha influido en esta población la medicalización de la fecundidad. La fuente de datos empleada es la Encuesta Demográfica Retrospectiva (EDER 2011). Con base en esta fuente de datos se analizan tres cohortes de nacimiento: 1951-1953, 1966-1968 y 1978-1980.

El contexto urbano en México, comparativamente con el rural, representa principalmente un factor que influye en reducir la fecundidad a través, primeramente, de ofrecer mayor acceso a los servicios públicos de salud, lo cual a su vez se traduce en mayor acceso a la anticoncepción moderna. Es necesario recordar que la disminución de la fecundidad inició en las ciudades y no fue sino 20 años después que se propagó a las zonas rurales (Zavala, 2004). Respecto a las especificidades de las mujeres indígenas que viven en el contexto urbano, cabe destacar que la mayoría ha tenido una experiencia migratoria que influye a su vez en la pérdida intergeneracional de la lengua indígena y en el calendario de formación de uniones. Se observan grandes desigualdades de género respecto a los hombres indígenas, principalmente en menor escolaridad y menor calificación en el empleo. Las parejas indígenas en las ciudades prefieren trayectorias 
normativas en la formación de sus familias en comparación con las no indígenas, quienes en ciertos grupos experimentan trayectorias innovadoras (Vázquez, 2016).

A escala nacional las mujeres hablantes de lengua indígena en comparación con las no hablantes de lengua indígena presentan una menor prevalencia de uso de métodos anticonceptivos; un menor acceso a los servicios de salud; unos niveles de fecundidad también menores al número promedio de hijos deseados, en un contexto de prácticas médicas asociadas al uso extensivo de la cesárea y proclives a sugerir el uso del DIU y la OTB. Todo lo anterior es consistente con la presunción que se busca comprobar en el presente artículo, de que las trayectorias medicalizadas de la fecundidad están más fuertemente asociadas con el declive de la descendencia en las mujeres indígenas que en el total de mujeres en un contexto urbano.

\section{ANTECEDENTES}

Durante las casi cinco décadas de las políticas de población antinatalista y de la difusión de programas de planificación familiar, el uso de métodos anticonceptivos en México se amplió masivamente a lo largo del país. Una de las formas de persuadir a la población para asumir u orientar los sentidos, creencias, valores y actitudes es mediante la transmisión de mensajes de medios de comunicación masiva (Sartori, 2002). Se ha confirmado en México que los mensajes de planificación familiar producidos por el Consejo Nacional de Población en 1980 estuvieron enfocados a la reducción de la familia tuvieron un impacto muy importante en la disminución de la fecundidad (Tuirán, 1996; Vázquez y Luna, 2000). Los spots de televisión y telenovelas promovían que la familia fuera más pequeña y que la pareja contara con estrategias de planificación familiar. En países como Brasil ocurrió algo muy similar, el acceso público a la televisora, que dominaba la transmisión de telenovelas introdujo un modelo de mujer independiente con pocos o sin ningún hijo, lo cual se relacionó con el rápido descenso de la fecundidad (La Ferrara et al., 2012).

La fecundidad en México sufrió cambios significativos a lo largo de las últimas seis décadas, la disminución de sus niveles se vio estrechamente relacionada con la expansión del uso de métodos anticonceptivos modernos. Sin embargo, los niveles de fecundidad han permanecido heterogéneos dentro del país, según características sociales como: el nivel de estudios alcanzado, lugar de residencia, el estado civil, la condición de identificación étnica o el uso de métodos anticonceptivos (CONAPO, 2016; Páez y Zavala, 2016). 
Puntualmente en México, la población indígena presenta una fecundidad más elevada en comparación con la población no indígena (Vázquez, 2010); aunque, las brechas van disminuyendo debido a que la reducción de la fecundidad en las mujeres hablantes de lengua indígena es más rápida que la de la no indígena a escala nacional. En las últimas décadas, la fecundidad a nivel nacional ha ido descendiendo gradualmente a causa de procesos como: el aumento del nivel educativo y la participación económica femenina. Sin embargo, entre las mujeres indígenas, esta reducción también se ha dado en todas las cohortes como consecuencia de la introducción y el ejercicio de los servicios de salud sexual y reproductiva en las zonas donde radican (Ham, 2003).

Efectivamente, desde una óptica transversal se observa que la brecha entre indígenas y no indígenas se va reduciendo con el pasar de los años. Las estimaciones muestran que la tasa global de fecundidad (TGF) para las mujeres hablantes de lengua indígena fueron de 4.1 hijos por mujer en 1999; 3.2 en 2009 y 3.0 en 2014, mientras que para las no hablantes de lengua indígena estos valores fueron de $2.7,2.3$ y 2.2 hijos por mujer respectivamente. La brecha de la TGF entre hablantes y no hablantes de lengua indígena pasa de 35 por ciento, en 1999, a 30 por ciento, en 2009, y 28, en 2014 (Vázquez y Cenobio, 2017).

Pareciera incluso que los niveles de fecundidad a escala nacional recientemente alcanzados fueran demasiado bajos, puesto que los datos de la Encuesta Nacional de la Dinámica Demográfica (ENADID) de 2014 señalan que en 2012 la TGF para las mujeres hablantes de lengua indígena eran de tres hijos por mujer, tasa mayor que para la población no indígena, que tiene un nivel muy cercano al reemplazo (2.17 hijos por mujer), estos datos son menores al ideal de 3.2 hijos que desea una mujer indígena en comparación con los 2.5 de una mujer no indígena (CONAPO, 2016). En otras palabras, las mujeres tienen menos hijos de los que desean tener.

Los niveles ligeramente más altos de fecundidad entre la población indígena están relacionados con distintos tipos de factores, entre los que se encuentran una unión y una maternidad más tempranas. En este sentido, resaltan las diferencias en la edad mediana a la formación familiar según condición de identificación étnica, las mujeres indígenas se unen o tienen su primer hijo alrededor de un año o dos antes en todos los grupos de edad de la etapa reproductiva (CONAPO, 2016).

Otros factores asociados a los niveles más altos de fecundidad indígena están relacionados con el menor conocimiento y uso de métodos anticonceptivos. Uno de los datos que más llama la atención es que 53.4 por ciento 
de estas mujeres no usaron métodos anticonceptivos en la primera relación sexual debido al desconocimiento de dónde obtener y cómo usarlos y 22.8 por ciento porque deseaban embarazarse. En contraste, estas mismas cifras para las mujeres no hablantes de lengua indígena es de 17.4 y 18.3 por ciento, respectivamente (CONAPO, 2016: 72).

Por lo que toca a los métodos de planificación familiar más usados, tanto por indígenas como por el total de las mujeres, sobresale el uso de la oclusión tubaria bilateral (OTB) o la esterilización femenina para limitar la descendencia y el uso del dispositivo intrauterino (DIU) para espaciar los nacimientos. Casi la mitad de las mujeres en edad fértil hablantes de lengua indígena y sexualmente activas ( 48.8 por ciento) es usuaria de OTB y 16.9 por ciento de DIU, mientras que, en las mujeres no hablantes de lengua indígena, el porcentaje de OTB es menor a 45 por ciento y de DIU de 16.6 por ciento (CONAPO, 2016).

Una variable relacionada con la disminución de la fecundidad y con el uso de métodos anticonceptivos como el DIU o la realización de la OTB es el tipo de parto, en específico la cesárea. Existe evidencia en Brasil de que ocurría violencia estructural en los programas de planificación familiar en los años ochenta, pues uno de sus objetivos era que trataban de reducir la población indígena mediante un proceso intensivo de esterilización, cuya aplicación a gran escala se dio en los hospitales públicos (Schooyans, 1997) y, algo muy parecido ocurrió en México con la población pobre (Barroso, 2004). Schooyans (1997) menciona que más de 72 por ciento las OBT en Brasil eran practicadas después de los nacimientos por cesárea y sin consentimiento de las mujeres.

En las últimas décadas, se ha incrementado la prescripción de la operación cesárea, sobrepasando indiscriminadamente la Norma Oficial Mexicana, con el fin de disminuir fallidamente la mortalidad materna (Aujang y Felguérez, 2009). Los motivos del uso masivo de la cesárea en el sistema de salud en el contexto mexicano son diversos y complejos.

Los intereses del cuerpo médico y las instituciones de salud para practicar los partos por cesárea son múltiples: logística de organización y rentabilidad de las infraestructuras, organización de horarios, remuneraciones mayores y protección contra denuncias, y percepción de los médicos de mayor control sobre el resultado (Rocha-Acero et al., 2019). Las mujeres pueden también optar por una cesárea para evitar el dolor, preservarse fisiológicamente de las consecuencias de un parto vaginal, planear la fecha del parto, o también porque su seguro cubre únicamente las cesáreas (Brugeilles, 2014a: 35). 
No obstante, el mito todavía prevaleciente en el ámbito médico de "una vez cesárea, siempre cesárea" es una de las principales indicaciones de esta operación (Secretaría de Salud, 2002a).

La medicalización ${ }^{1}$ de la vida reproductiva de las mujeres se ha radicalizado con el aumento de cesáreas y esterilizaciones que han ayudado a la disminución de la fecundidad (Brugeilles, 2014b). La medicalización es el gran contexto en el que ocurre la violencia obstétrica y el uso abusivo de la esterilización femenina (Salgado y Díaz, 2019). El cuerpo es una construcción social derivada de la medicalización impulsada, a su vez, por la tecnología, las farmacéuticas, la ideología dominante del personal médico y el mercado. (Salgado y Díaz, 2019). Se entiende por violencia obstétrica:

La apropiación del cuerpo y procesos reproductivos de las mujeres por prestadores de salud, que se expresa en un trato jerárquico deshumanizador, en un abuso de medicalización y patologización de los procesos naturales, trayendo consigo pérdida de autonomía y capacidad de decidir libremente sobre sus cuerpos y sexualidad impactando negativamente en la calidad de vida de las mujeres (Gaceta Oficial de la República Bolivariana de Venezuela, 2007).

Una de las expresiones teóricamente mejor articuladas para explicar la violencia obstétrica y el autoritarismo con que en muchas ocasiones son tratadas las mujeres en los hospitales en México, se concreta en la categoría de habitus médico (Castro, 2014). El habitus médico:

...es el conjunto de predisposiciones generativas que resultan de la incorporación (lo social hecho cuerpo) de las estructuras objetivas del campo médico. Tales predisposiciones las adquieren los profesionales de este campo - en primer lugar, los médicos - a través de la formación que reciben en la facultad de medicina y en los hospitales de enseñanza (Castro, 2014: 173).

El campo médico son las instituciones de salud y las instituciones de formación de médicos, tales como facultades de medicina y los hospitales de enseñanza. Considerando lo anterior, el habitus médico dentro del campo médico genera un sentido práctico, el cual constituye un actuar preconsciente por parte del médico que es funcional para los fines de las instituciones de salud (el campo médico en su conjunto). El punto clave en este enfoque sociológico consiste, desde nuestro punto de vista, en explicar el

\footnotetext{
${ }^{1}$ Se entiende por medicalización como "la forma en que el ámbito de la medicina moderna se ha expandido en los años recientes y ahora abarca muchos problemas que antes no eran considerados como entidades médicas" (Aguilar, 2015: 262). En parte, la medicalización implica la supervisión, diagnóstico y tratamiento desde la "mirada médica" de personas no enfermas durante el transcurso de eventos normales de la vida reproductiva como es el embarazo, el parto y el control del tamaño de la descendencia.
} 
vínculo causal existente entre el autoritarismo que exhiben en su práctica profesional los médicos como consecuencia del conjunto de mensajes, enseñanzas, conminaciones, recriminaciones, clasificaciones, etiquetaciones, descalificaciones, regulaciones y jerarquizaciones que reciben durante sus estudios. De esta manera, el habitus médico es una causa estructural de las violaciones a los derechos de las mujeres, y es muy distinto y de mayor repercusión al discurso médico hegemónico que las relega la "calidad" de los servicios.

Este sentido práctico del personal médico, no hay que olvidarlo, se vincula al mismo tiempo con el contexto de una política nacional de población, una política transexenal y de Estado, instrumentada principalmente por el sector salud, que por muchos años privilegió la eficacia de las prácticas y de los métodos para alcanzar las metas del descenso de la fecundidad. Esta conjunción alimentó valores, preceptos, "buenas intenciones", que entre otras cosas privilegiaron la decisión autorizada del médico en materia reproductiva sobre la decisión de las mujeres en el control de sus propios cuerpos.

Además, se ha podido relacionar el conocimiento de los riesgos de mortalidad y morbilidad materna provocados por la cesárea para infundir la aceptación de métodos anticonceptivos definitivos como la OTB o más efectivos como el DIU, que con el paso del tiempo han ayudado a la disminución de la tasa de fecundidad (Aujang y Felguérez, 2009; Lara-Contreras et al., 2018). Técnicamente hablando, tanto el DIU como la OTB pueden aplicarse en el posparto, transcesárea y posaborto, pero la literatura médica en México recomienda ser aplicado antes de que la usuaria abandone la unidad médica. Ambos procedimientos pueden ser realizados durante la operación cesárea a través de histerotomía (Secretaría de Salud, 2002b: 1).

A nivel nacional, el porcentaje de aceptación del dispositivo intrauterino y la OTB durante la operación cesárea ha aumentado en el mismo sentido en el periodo de 1990 y el 2010. Es decir, que se va observando una correlación positiva entre la operación cesárea y el uso o aceptación del DIU y en mayor medida de la OTB. Diversos estudios también arrojan que la aceptación de la esterilización está más relacionada con la cesárea (Brugeilles, 2014b) y el dispositivo intrauterino cuenta con mayor aceptación durante el parto vaginal (Aujang y Felguérez, 2009). El sector salud "recomienda promover que toda paciente sometida a operación cesárea deberá egresar del hospital con un método temporal o definitivo de anticoncepción" (IMSS, 2014:14). 
Aunque se supone que existe un acceso al sistema de salud y a la medicalización relacionada con la procreación, es conocido que la población indígena y rural utiliza en menor medida estos servicios, lo cual está relacionado con factores culturales, sociales, económicos y religiosos (Celi, 2018). A raíz de que la medicalización relacionada con la procreación se introdujo de una manera eficiente, donde los agentes de salud están en una posición más privilegiada que las mujeres, esto les permite difundir ideales de fecundidad, incluyendo el lugar de atención del parto y el tipo de parto (Lerner et al., 1994).

Es sabido que, en México, desde la puesta en práctica de los programas de planificación familiar, el lugar de atención del parto es un determinante del uso de métodos anticonceptivos (Lerner et al., 1994). Las mujeres no usuarias que atienden sus partos en hospitales o clínicas de salud salen del estos sobre todo con el DIU o la operación femenina. Esto es el resultado esperado de las políticas de población aplicadas por el sector salud basado en acciones de consejería dirigidas a incrementar los indicadores de nuevas aceptantes haciendo uso de las oportunidades disponibles. Por contraparte, las mujeres que dieron a luz a sus hijos en domicilios particulares están en cierta medida protegidas del riesgo de usar métodos controlados por médicos ya fuera por cuestiones culturales, por falta de acceso a los servicios de salud o por la resistencia premeditada para evitar ser tratadas en un contexto medicalizado que intimida y atemoriza a algunas de ellas.

\section{Metodología Y Datos}

La fuente de datos empleada es la Encuesta Demográfica Retrospectiva (EDER 2011), la cual es de cobertura nacional de la población residente en un área urbana de México al momento de la entrevista con base en una muestra de 3,200 viviendas. Con base en esta fuente de datos se analizan tres cohortes de nacimiento: 1951-1953, 1966-1968 y 1978-1980. La identificación indígena fue construida a partir de tres categorías: la condición de hablante de lengua indígena, el origen indígena (es decir, ser hija de padre y/o madre hablante de lengua indígena) y la pertenencia a un pueblo indígena por autoadscripción. Si la mujer respondió que sí al menos de una de estas tres categorías es reconocida como indígena (Vázquez, 2016). Para su comparación, se analizará al conjunto de la población femenina total entrevistada residente en zonas urbanas (incluidas las propias indígenas). A lo largo del texto, para abreviar, se referirá a ambos grupos como "indígenas" y "total de las mujeres". En términos absolutos, la muestra contiene 157 mujeres indígenas y 1,296 total de mujeres, lo que se traduce 
en 7,276 y 58,423 años persona, respectivamente, aplicados al análisis de sobrevivencia.

Para aclarar posibles dudas, cabe mencionar que al interior de la categoría de identificación indígena se encuentra el total de las mujeres hablantes de lengua indígena, así como el total de las que tienen origen indígena y el total de las que pertenecen a un pueblo indígena. Por lo tanto, si bien referimos en párrafos anteriores de este mismo documento la categoría "hablantes de lengua indígena", esto se debe simplemente a que este es identificador étnico en las fuentes citadas. La categoría "indígena" que se empleará en lo sucesivo, tiene varias mejoras que complementan a la de "hablante de lengua indígena", a saber: que la pertenencia refiere a un pueblo indígena, más apegada al enfoque de derechos que aplica a pueblos y comunidades, y no al de una cultura indígena; que en la EDER de 2011 la pregunta sobre pertenencia étnica se formula directamente sobre sí mismo y no respecto a otros miembros del hogar, como se hace en la encuestas de los censos de México a partir del año 2000; pero una mejora sustancial es que, al incluir a los individuos cuyos padres son (o fueron) hablantes de lengua indígena se incluye a los individuos indígenas que por la pérdida de la transmisión intergeneracional de la lengua ya no hablan su lengua. Este último caso se encuentra sobrerrepresentado en el medio urbano, producto de la migración campo-ciudad, por lo que la categoría de identificación étnica tal como está construida es especialmente adecuada para este trabajo.

Con el propósito de analizar si las características relacionadas con el nacimiento del hijo anterior están relacionadas con el siguiente ajustamos dos modelos estadísticos de análisis de supervivencia de tiempo discreto (Allison, 2014). En estos modelos se divide el ciclo de vida de las personas en episodios, en este caso de un año. Estos segmentos anuales son utilizados como observaciones o casos en un modelo logístico, en donde la variable dependiente es dicotómica y diferencia entre haber experimentado el evento, por ejemplo, nacimiento del segundo hijo (categoría $=1$ ) o cuando no se ha experimentado (categoría $=0$ ).

Denotamos el riesgo por $\mathrm{P}(\mathrm{t})$, la probabilidad condicional de que una de nuestras mujeres tenga un evento en el tiempo $t$, dado que ésta no ha presentado todavía el evento.

$$
\mathrm{P}(\mathrm{t})=\mathrm{b}_{0}+\mathrm{b}_{1} \mathrm{x}_{1}+\ldots \mathrm{b}_{\mathrm{n}} \mathrm{x}_{\mathrm{n}}
$$

En este paso especificamos como el riesgo de tener un siguiente hijo depende de las variables exploratorias. En otras palabras, para analizar el nacimiento del segundo hijo ajustamos el modelo con variables: tipo de 
parto, lugar de atención durante el primer parto y el último método anticonceptivo (Tabla 1) y para la transición al tercer hijo nos apoyamos de las variables relacionadas con el nacimiento del segundo hijo. Además, se añade como variable exploratoria la cohorte de nacimiento, con el objetivo de mostrar las diferencias entre mujeres por esta variable.

Tabla 1: Descripción de las variables en los modelos

\begin{tabular}{|c|c|}
\hline Variable & Descripción \\
\hline \multicolumn{2}{|c|}{ Modelo: Transición entre el primer y el segundo hijo } \\
\hline $\begin{array}{l}\text { Condición de identificación } \\
\text { indígena }\end{array}$ & 1, Total; 2, Indígena \\
\hline $\begin{array}{l}\text { Último método anticonceptivo } \\
\text { usado }\end{array}$ & $\begin{array}{l}\text { 1, Diu; 2, No uso; 3, Tradicionales; } \\
\text { 4, Condón; 5, Hormonales (Pastillas, } \\
\text { Inyecciones y Norplant) }\end{array}$ \\
\hline Tipo de parto del primer nacimiento & 1, Vaginal; 2, Cesárea \\
\hline $\begin{array}{l}\text { Lugar de atención del primer } \\
\text { nacimiento }\end{array}$ & $\begin{array}{l}\text { 1, Hospital (Incluye: Seguro social, } \\
\text { hospital público y otra institución de } \\
\text { gobierno, consultorio, clínica u hospital } \\
\text { privado); 2, Casa de la mujer; 3, Otro }\end{array}$ \\
\hline $\begin{array}{l}\text { Duración entre el primer y segundo } \\
\text { nacimiento }\end{array}$ & $\begin{array}{l}\text { Tiempo en años que transcurrió entre } \\
\text { nacimientos }\end{array}$ \\
\hline \multicolumn{2}{|c|}{ Modelo: Transición entre el segundo y el tercer hijo } \\
\hline Identificación indígena & 1, No indígena; 2 , Indígena \\
\hline $\begin{array}{l}\text { Último método anticonceptivo } \\
\text { usado }\end{array}$ & $\begin{array}{l}\text { 1, Diu; 2, No uso; 3, Tradicionales; } \\
\text { 4, Condón; 5, Hormonales (Pastillas, } \\
\text { Inyecciones y Norplant); 6, OBT }\end{array}$ \\
\hline Tipo de parto del primer nacimiento & 1, Vaginal; 2, Cesárea \\
\hline $\begin{array}{l}\text { Lugar de atención del primer } \\
\text { nacimiento }\end{array}$ & $\begin{array}{l}\text { 1, Hospital (Incluye: Seguro social, } \\
\text { hospital público y otra institución de } \\
\text { gobierno, consultorio, clínica u hospital } \\
\text { privado); 2, Casa de la mujer; } 3 \text {, Otro }\end{array}$ \\
\hline $\begin{array}{l}\text { Duración entre el primer y segundo } \\
\text { nacimiento }\end{array}$ & $\begin{array}{l}\text { Tiempo en años que transcurrió entre } \\
\text { nacimientos }\end{array}$ \\
\hline Cohorte de nacimiento & $\begin{array}{l}\text { 1, 1951-1953; 2, 1966-1968; 3, 1978- } \\
1980\end{array}$ \\
\hline
\end{tabular}

Fuente: elaboración de los autores.

En el caso de esta transición incluimos en la variable método anticonceptivo actual usando la categoría OBT, ya que el porcentaje de opera- 
ciones femeninas para el tercer hijo aumenta considerablemente. Lo cual mostrará que esta intervención no es totalmente eficaz para limitar los nacimientos. Para interpretar de una forma más eficiente los resultados de los modelos calculamos la función de supervivencia y la duración mediana de cada grupo de mujeres.

\section{RESULTADOS}

\section{Fecundidad y uso de métodos anticonceptivos modernos}

Los datos de la EDER de 2011 corroboran que la fecundidad de las mujeres indígenas urbanas se encuentra en descenso, lo cual se aprecia en que la descendencia alcanzada a los 30 años pasó de 3.3 a 2.5 y a 2.0 hijos por mujer para las cohortes avanzada, intermedia y joven respectivamente. La cohorte intermedia de mujeres indígenas es la que presenta un descenso mayor en comparación con la más joven, lo cual es extraño, ya que la cohorte intermedia sufrió un leve descenso en la proporción de usuarias de métodos anticonceptivos modernos. La proporción de usuarias de estos métodos a los 30 años es de 45, 43 y 51 por ciento para las cohortes 19511953, 1966-1968 y 1978-1980, respectivamente. Cabe mencionar que considerando la aleatoriedad de los datos debidos al tamaño reducido de la muestra estas proporciones son resultado de una estimación suavizada mediante la técnica de las medias móviles.

Dada esta información se puede hacer la siguiente pregunta: ¿Por qué las mujeres indígenas de las generaciones intermedias disminuyeron su fecundidad aceleradamente mientras que presentaron un descenso en la prevalencia de uso de métodos anticonceptivos? Una suposición puede ser debido al uso de métodos anticonceptivos más efectivos por parte de esta cohorte. La evidencia señala que ciertamente las mujeres indígenas de la cohorte intermedia incrementaron en proporción el uso de la OTB y el DIU y rejuvenecieron notablemente el calendario de la OTB, como se detalla a continuación. La proporción de mujeres usuarias de OTB con al menos un hijo observadas hasta los 30 años, para el total de las tres cohortes, es mayor para las indígenas que para el conjunto de mujeres entrevistadas: 26 y 21 por ciento, respectivamente.

Por otra parte, el análisis por cohortes de nacimiento evidencia que la proporción del uso de OTB se va incrementando a medida que se consideran cohortes más jóvenes tanto indígenas como para el total de las mujeres, y en ambos casos el incremento en la cohorte más joven es apenas de uno o dos puntos porcentuales. En la población indígena la proporción casi se 
triplica en la cohorte intermedia respecto a la cohorte avanzada, mientras que en la población total casi se duplica. En las cohortes intermedia y joven la proporción de usuarias de OTB es superior en las indígenas respecto al promedio del total de mujeres: para las indígenas es $11.7,29.5$ y 31.5 por ciento mientras que en el total femenino es $13.6,22.4$ y 23.4 por ciento para las cohortes avanzada, intermedia y joven respectivamente.

Otro factor en el descenso de la fecundidad de las mujeres indígenas de la cohorte intermedia es el calendario del uso de la OTB de las mujeres analizadas con al menos un hijo, el cual se ha ido rejuveneciendo. La edad mediana a la realización de la OTB para el total de mujeres entrevistadas ha pasado de 32 a 31 y 27 años en las cohortes avanzada, intermedia y joven; en las mujeres indígenas las edades son 33, 27 y 27 años respectivamente. Mientras el rejuvenecimiento es más notorio en la cohorte más joven para el total de mujeres, en las indígenas se aprecia una caída abrupta en la cohorte intermedia cuya edad mediana a la OTB ocurre seis años antes que en la cohorte avanzada y cuatro años antes que en el total de las mujeres de su propia cohorte.

Por lo que toca a la proporción de mujeres usuarias por lo menos una vez en la vida de DIU con al menos un hijo hasta los 30 años, se observa que esta es 30 por ciento para todas las mujeres entrevistadas y 28 por ciento para las indígenas. Por cohortes de nacimiento la proporción del uso del DIU se va incrementando a medida que se consideran cohortes más jóvenes en indígenas y para el total. En las mujeres indígenas las proporciones de usuarias son $11.1,32.5$ y 33.3 por ciento mientras que en el total de las mujeres son $18.1,29.8$ y 36.5 por ciento para las cohortes avanzada, intermedia y joven, respectivamente. Una vez más, mientras el incremento es relativamente paulatino en las mujeres del país, en las indígenas ocurre un incremento muy importante en la cohorte intermedia, la cual casi triplica la proporción de la cohorte avanzada y supera al total de las mujeres de su propia cohorte, y después un incremento mucho menor en la cohorte joven. El calendario del primer DIU se rejuvenece en el total de mujeres, pero en cambio en las indígenas se retrasa en la cohorte intermedia y se rejuvenece en la más joven. Las edades medianas para estas mujeres son 24, 22 y 21 años en las cohortes avanzada, intermedia y joven y para las indígenas de 20, 22 y 21 años respectivamente. Ham (2003) reconoce que, en términos generales, la reducción de la fecundidad indígena recae en la utilización del DIU o la terminación del periodo reproductivo a través de la OBT, una vez que se ha tenido el tamaño de familia deseado. 


\section{Lugar del parto}

La proporción de partos atendidos en una unidad médica del sector público (Instituto Mexicano del Seguro Social, IMSS, Instituto de Seguridad y Servicios Sociales de los Trabajadores del Estado, ISSSTE, Secretaria de Salud, SSA, Sistema Nacional para el Desarrollo Integral de la Familia, DIF) $\mathrm{u}$ otra institución de gobierno federal o estatal es un indicador de acceso a los servicios de salud materna y de medicalización del parto. Este indicador confirma la tendencia al aumento tanto en la población indígena, al pasar de 44 a 53 y 73 por ciento para las cohortes avanzada, intermedia y joven respectivamente, como en el total de mujeres entrevistadas, con 59, 68 y 73 por ciento (Tabla 2). La brecha entre las mujeres que atendieron sus partos en un servicio de salud pública entre mujeres indígenas y nacional fue de 17 puntos porcentuales en las cohortes avanzada e intermedia y se reduce a cero abruptamente en la cohorte más joven.

Tabla 2: Lugar de nacimiento de los hijos de las mujeres según condición de identificación étnica y cohorte (en porcentaje)

\begin{tabular}{lrrrrr}
\hline $\begin{array}{l}\text { Cohorte de } \\
\text { nacimiento }\end{array}$ & $\begin{array}{r}\text { Servicio } \\
\text { público }\end{array}$ & $\begin{array}{r}\text { Servicio } \\
\text { privado }\end{array}$ & Domicilio & Otro & Suma \\
\hline $\begin{array}{l}\text { Mujeres indígenas } \\
1951-1953\end{array}$ & 44.0 & 13.0 & 43.0 & 0 & 100.0 \\
$1966-1968$ & 53.0 & 17.0 & 30.0 & 0 & 100.0 \\
$1978-1980$ & 73.0 & 18 & 8.0 & 0 & 100.0 \\
Total de mujeres & & & & & \\
$1951-1953$ & 59.0 & 19.0 & 23.0 & 0 & 100.0 \\
$1966-1968$ & 68.0 & 22.0 & 9.0 & 0 & 100.0 \\
$1978-1980$ & 73.0 & 23.0 & 3.0 & 1 & 100.0 \\
\hline
\end{tabular}

Fuente: cálculos propios con base en la EDER-2011.

La ocurrencia del parto en el propio domicilio de las madres o en el de las parteras, que ha sido un rasgo característico del comportamiento reproductivo de las mujeres indígenas en nuestro país, muestra un descenso importante en la cohorte intermedia, pero mucho más significativo en la cohorte más joven. Pese a su reducido tamaño, la proporción de partos en domicilio es casi cuatro veces mayor en las indígenas respecto al total de mujeres (ocho y tres por ciento, respectivamente) en la cohorte más joven. Finalmente, el sector privado muestra un ligero incremento en la propor- 
ción de partos atendidos a medida que se consideran cohortes de mujeres más jóvenes, este incremento es más modesto entre las mujeres indígenas.

\section{Tipo de parto}

La cesárea frente al parto vaginal se ha incrementado muy notablemente a nivel nacional a medida que se consideran generaciones más jóvenes, tal como lo han mostrado Brugeilles y Rojas (2016) con base en la EDER 2011.

En las tres cohortes, las indígenas son quienes presentan una proporción más alta de cesáreas y, a medida que se considera a las cohortes más jóvenes esta característica se acentúa. En la cohorte avanzada la diferencia entre la proporción de mujeres que presentan una o más cesárea entre los 15 y los 30 años es muy reducida, de apenas un punto porcentual; pero esta diferencia se incrementa a seis y ocho puntos porcentuales en las cohorte intermedia y joven respectivamente (Tabla 3 ). Nuevamente la cohorte intermedia en las mujeres indígenas presenta un incremento abrupto en la proporción de intervenciones médicas asociadas a la maternidad, como es la cesárea, respecto a la cohorte avanzada.

Tabla 3: Proporción de mujeres con al menos un hijo según realización de una o más cesáreas hasta los 30 años de edad por condición de identificación étnica y cohorte (en porcentaje)

\begin{tabular}{lccc}
\hline Identificación étnica & No & Sí & Total \\
\hline Cohorte 1951-1953 & 86.0 & 14.0 & \\
Total & 85.0 & 15.0 & 100.0 \\
Indígena & & & 100.0 \\
Cohorte 1966-1968 & 69.0 & 31.0 & 100.0 \\
Total & 63.0 & 37.0 & 100.0 \\
Indígena & & & \\
Cohorte 1978-1980 & 65.0 & 35.0 & 100.0 \\
Total & 57.0 & 43.0 & 100.0 \\
Indígena &
\end{tabular}

Fuente: cálculos propios con base en la EDER-2011.

Se ha visto que las mujeres indígenas tienen un menor acceso al sistema de salud, pero entonces, ¿Por qué tienen una mayor proporción de cesárea que para el total de mujeres, que son intervenciones 100 por ciento hospita- 
larias? No podemos ofrecer una respuesta con los datos de la EDER 2011, pero es poco probable que las causas estrictamente médicas expliquen la mayor proporción de cesáreas en las indígenas, por lo que la respuesta deberá buscarse en las decisiones de las mujeres y en la influencia del entorno médico.

En la cohorte avanzada, la cesárea en las mujeres indígenas con hijos presenta proporciones muy inferiores a todo lo largo de la vida reproductiva respecto al total de las mujeres entrevistadas. En cambio, en la cohorte intermedia las proporciones de cesáreas son muy similares entre ambos. Finalmente, en la más joven las proporciones de cesárea en las mujeres indígenas supera a la de las mujeres en el país, prácticamente en todas las edades de la vida reproductiva. Es de llamar la atención que el caso de la cohorte más joven presenta una distribución en forma de $U$, donde las niñas y adolescentes con hijos alcanzan valores cercanos a 60 por ciento de cesáreas, después desciende al llegar de los 18 a los 22 años, para después volver a aumentar, siempre con los valores más elevados registrados en las cohortes anteriores.

Esta distribución en forma de U es anómala respecto a las cohortes anteriores, puesto que todas ellas empezaban con bajos promedios en las edades correspondientes a la niñez y adolescencia. Además, se repite en la población femenina total y en la indígena, por lo que puede ser resultado de una intencionalidad expresa de atención por cesárea a los partos en las mujeres a edades muy jóvenes (Figura 1), sin embargo, esto ocurrió antes de 2015, año en que el gobierno federal pusiera en operación la Estrategia Nacional para la Prevención del Embarazo en Adolescentes (ENAPEA) por lo que no puede ser atribuible a dicha política.

\section{Trayectorias relacionadas con la vida reproductiva}

En este apartado se reconstruyen y analizan las trayectorias del ciclo reproductivo a partir de tres eventos, el nacimiento del primer, el segundo y el tercer hijo, diferenciando tres situaciones: por el tipo de lugar donde ha ocurrido el nacimiento (hospital o casa de la mujer), por el tipo de parto (vía vaginal o por cesárea) y finalmente si se adoptado algún método anticonceptivo (DIU u OBT) o ninguno.

Así, para cada paridad primeramente de detectaron 24 trayectorias; sin embargo, algunas de ellas resultaron una combinación de eventos sin sentido para el análisis y además pulverizaron el tamaño de muestra, por lo que se optó por agruparlas en siete trayectorias más robustas y de interés, las cuales se presentan de forma ascendente según su grado de medicalización (Tabla 4). 
Figura 1: Proporción de cesáreas en indígenas y total de mujeres con al menos un hijo (las series indígenas suavizadas por medias móviles)

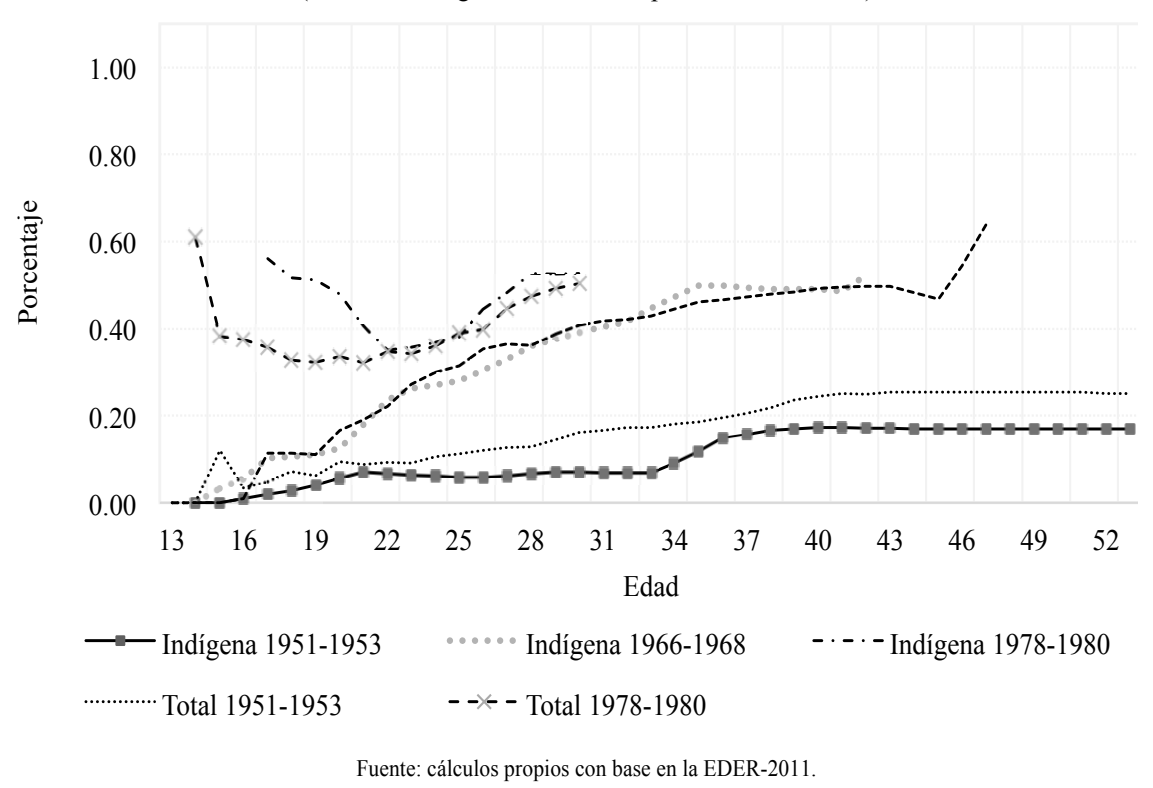

Lo primero a destacar es que, independiente del orden de nacimiento de los hijos y de la situación de ser indígena o de pertenecer al total de mujeres, se cumplen tres características generales de las trayectorias de la vida reproductiva, a saber: las trayectorias de una vida reproductiva más medicalizada, que son aquellas que inician con la atención del parto en hospitales por cesárea, terminan más frecuentemente con el uso del DIU o la OTB.

Esta situación se agudiza con los hijos de orden dos y sobre todo con los de orden tres donde los nacimientos casi no finalizan en no uso de métodos; i) las trayectorias que inician con el nacimiento de un hijo en un hospital pero éste ocurre vía vaginal en vez de cesárea, tienen como desenlace típicamente el uso de la OTB o el DIU, y; ii) Las trayectorias menos medicalizadas, que son aquellas que inician con el parto en casa y por vía vaginal y tienen la misma probabilidad de desembocar en el uso del DIU, de la OTB o del no uso de métodos. El uso del DIU es más frecuente después del primer hijo y la OTB después del segundo y del tercero. 
Planificación familiar y fecundidad de la población indígena en el México urbano / G. VÁZQUEZ SANDRIN y E. ORTíz ÁVILA

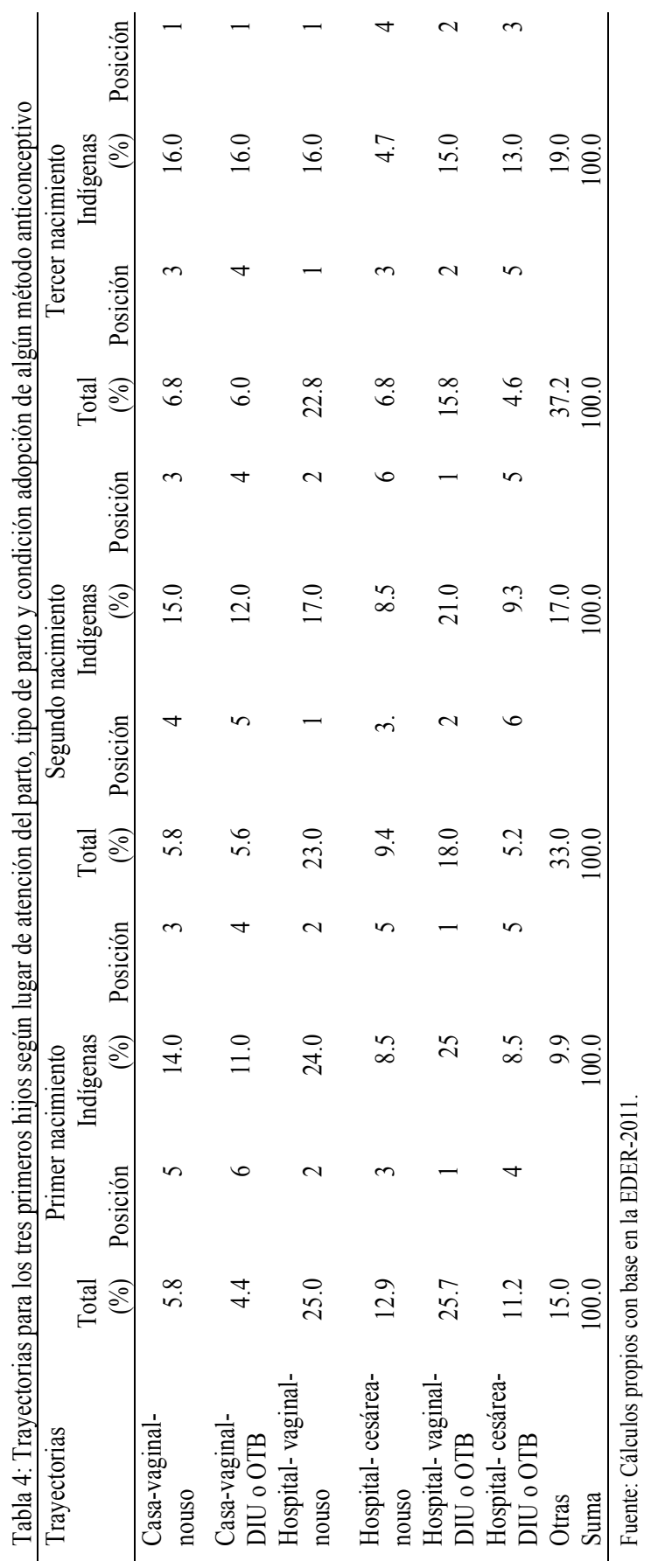


En cuanto a la diferencia entre indígenas y el total de mujeres, el análisis de trayectorias permite inferir que la asociación existente entre el grado de medicalización y el uso de métodos como el DIU o la OTB opera de forma similar para ambas poblaciones. Las diferencias entre ellas estriban en la frecuencia en que cursan las distintas trayectorias más o menos medicalizadas. Al ordenarlas por importancia en cada grupo y paridad de la madre, se observa que al primer nacimiento las tres trayectorias más frecuentes entre el total de mujeres son: nacimiento en hospital por vía vaginal con la utilización del DIU u OTB (hospital-vaginal-DIU u OBT), seguido de atención del nacimiento en hospital, parto vaginal y no uso de ningún método anticonceptivo después (hospital-vaginal-no uso), y en tercer lugar nacimiento en hospital, parto por cesárea y no uso de método (hospital-cesárea-no uso). En cambio, para las indígenas en los dos primeros solo se cambia en orden y el tercero es el mismo. En ambos casos se presenta la utilización del DIU u OBT como lo indica la recomendación de la Secretaria de Salud (2002b), la cual menciona que estos métodos se deben aplicar antes del egreso hospitalario. Para el segundo nacimiento, las tres trayectorias más comunes en el total de mujeres vuelven a ser las mismas que con el primer hijo y en las indígenas también salvo que en ellas muy cerca del tercer lugar se encuentra casa-vaginal-no uso. En el tercer hijo del total de las mujeres repiten los tres primeros lugares al igual que en su primer y segundo hijos, incluyendo en el tercer lugar también a casa-vaginal-no uso. En las indígenas el primer lugar es de tres categorías: casa-vaginal-no uso, casa-vaginal-DIU u OTB y hospital-vaginal-no uso, para el segundo y tercer lugar se tiene: hospital-vaginal-DIU u OTB y hospital-cesárea-DIU u OTB. En las trayectorias reproductivas de las mujeres indígenas es menos frecuente que esté involucrada una cesárea que en el total de mujeres. Sin embargo, sea que estas mujeres indígenas tengan el parto en un hospital o en una casa, que sea vaginal o cesárea, es más frecuente que su trayectoria termine con la OTB.

\section{Efecto de variables asociadas al nacimiento anterior en el descenso de la descendencia}

Se puede observar en la Tabla 5 los resultados del ajuste del primer modelo donde se pretende conocer el efecto de las variables relacionadas con el nacimiento del primer hijo para experimentar un segundo nacimiento. El primer resultado que llama la atención es en cuanto a la variable condición de identificación étnica. Este factor no es significativo, lo cual nos señala que no hay diferencias reveladoras entre las indígenas y el total de mujeres 
en la transición al siguiente hijo. Es decir, que en la transición al segundo las mujeres indígenas no son distintas de las indígenas. Las únicas categorías significativas de uso de métodos anticonceptivos son el DIU y el no uso. Lo que significa que las otras categorías no son distintas a la categoría de referencia, en este caso el DIU. La variable tipo de parto y lugar de atención también fueron significativas, excepto la categoría otro lugar de atención.

Tabla 5: Razón de momios de los modelos logísticos

\begin{tabular}{lrr}
\hline $\begin{array}{l}\text { Variable } \\
\text { Categoría }\end{array}$ & $\begin{array}{r}\text { Primer - segundo hijo } \\
\text { Exp (B) }\end{array}$ & $\begin{array}{r}\text { Segundo - Tercer hijo } \\
\text { Exp (B) }\end{array}$ \\
\hline Último método anticonceptivo & & \\
DIU & Ref. & Ref. \\
No uso & 1.170 & $1.587^{*}$ \\
Tradicionales & 0.941 & 1.044 \\
Condón & 1.058 & 0.886 \\
Hormonales & 1.094 & 1.079 \\
OBT & - & $1.388^{*}$ \\
Tipo de parto en el nacimiento del último hijo & \\
Vaginal & Ref. & Ref. \\
Cesárea & $0.628^{* * *}$ & $0.466^{*}$ \\
Lugar de atención del último parto & & \\
Hospital o clínica & Ref. & Ref. \\
Casa de la mujer & $1.290^{* *}$ & $1.795^{*}$ \\
Otro & 0.689 & 0.275 \\
Identificación indigena & & \\
Total & Ref. & Ref. \\
Indígena & 1.035 & 1.152 \\
Cohorte de nacimiento & & \\
1951-1953 & Ref. & Ref. \\
1966-1968 & $0.758^{* *}$ & $0.709^{*}$ \\
1978-1980 & $0.646^{* * *}$ & $0.758^{*}$ \\
\hline Sign & &
\end{tabular}

Significancia estadística: *** $0.01, * * 0.05$ y $* 0.10$.

Fuente: Cálculos propios con base en la EDER-2011. 
Para resumir los resultados del modelo "Transición entre el primer y el segundo hijo" presentamos la función de supervivencia de cuatro grupos de mujeres (Figura 2). En la figura observamos que, una vez efectuada la transición al primer hijo, una proporción importante de mujeres que usaron el DIU después del primer parto, que tuvieron su primer hijo por vía vaginal y que tuvieron ese hijo en casa de la mujer, tienen un segundo hijo. Esta proporción aumenta considerablemente a partir del tercer año en las tres cohortes. Las otras situaciones parecen producir un retraso a la transición del segundo hijo, pero en la que se percibe un mayor retraso y una menor proporción de entrada es en la transición de las mujeres que utilizan el DIU, el tipo de parto fue por cesárea y la atención fue en un hospital. Comparando con la situación anterior observamos que en la duración de tres años casi 50 por ciento de las mujeres no ha llevado a cabo esta transición en las tres cohortes.

La mediana de la duración nos muestra importantes diferencias entre grupos de mujeres, puesto que este último grupo mencionado (Diu-hospital-cesárea) tiene una mediana en la duración de 2.5 años entre el primer y segundo hijo, mientras que por parto vaginal (Diu-hospital-vaginal) es de casi la mitad (1.5 años). Las duraciones medianas más cortas las observamos en la cohorte avanzada y la intermedia y, las más largas en la cohorte más joven. Finalmente, podemos decir que ser usuaria del DIU, tener un parto por cesárea y en un hospital durante el primer parto son factores que disminuyen la probabilidad de tener un segundo hijo.

En la Tabla 5 podemos observar que la variable de identificación étnica en la transición del segundo al tercer hijo tampoco es significativa, por lo que al menos hasta el tercer hijo esta no es una variable que cause diferencias en cuanto a la fecundidad en estas dos poblaciones. La variable cohorte de nacimiento sí es significativa y demuestra que existe una menor probabilidad de tener un tercer hijo para las dos generaciones más jóvenes con respecto a la más antigua.

Del mismo modo presentamos la función de supervivencia para resumir los resultados del modelo "Transición entre el segundo y el tercer hijo" de ocho grupos de mujeres por cohorte de nacimiento (Figura 3). En estas gráficas se puede observar por cohorte de nacimiento un mismo patrón por grupo de mujeres. 
Planificación familiar y fecundidad de la población indígena en el México urbano / G. VÁZQUEZ SANDRIN y E. ORTíz ÁVILA

Figura 2: Transición del primero al segundo hijo según grupo de mujeres

Cohorte 1951-53

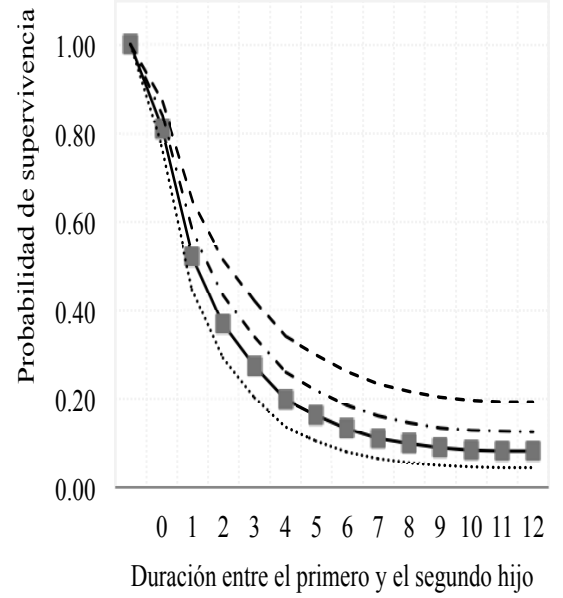

Cohorte 1966-68

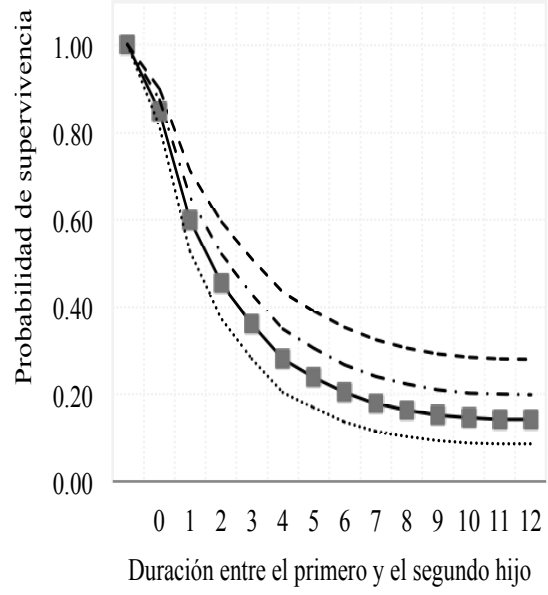

\footnotetext{
- DIU-vaginal-hospital ………. DIU-vaginal-casa

- - - - DIU-cesárea-hospital - - - - DIU-cesárea-casa
}

- D DIU-vaginal-hospital ……… DIU-vaginal-casa

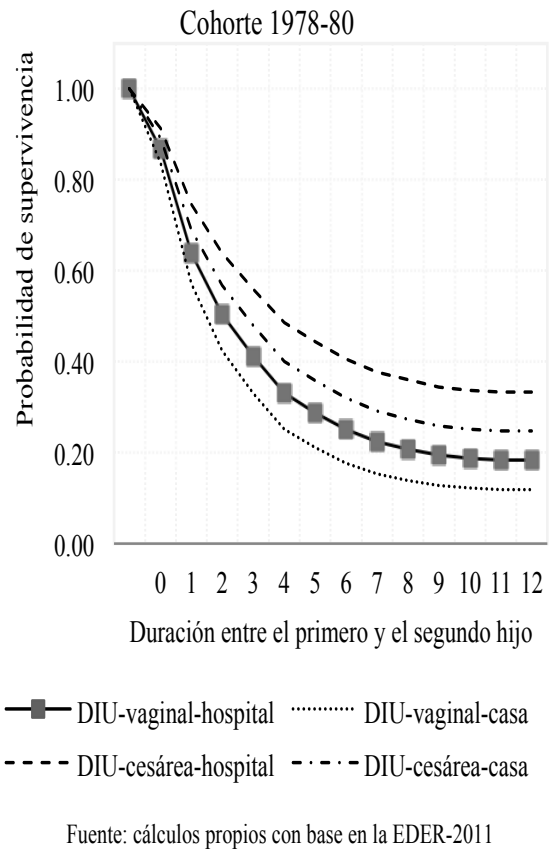


Figura 3: Transición del segundo al tercer hijo por cohorte de nacimiento según grupo de mujeres

Cohorte 1951-53

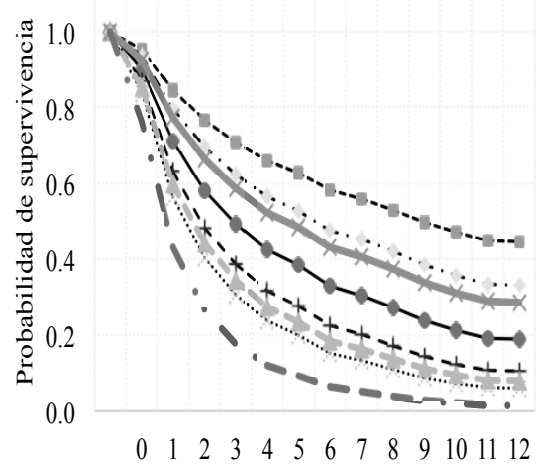

Duración en años entre el segundo y tercer hijo
Cohorte 1966-68

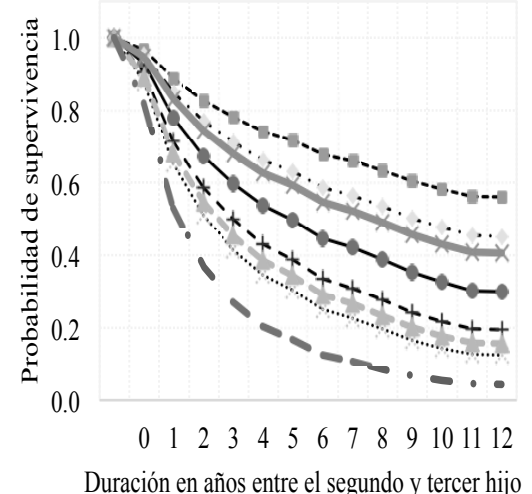

Duración en años entre el segundo y tercer hijo

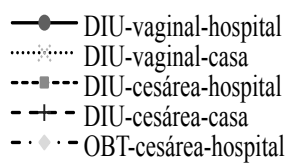

- D DIU-vaginal-hospital

---1---D DIU-cesárea-hospital

-. - OBT-cesárea-hospital

Cohorte 1978-80

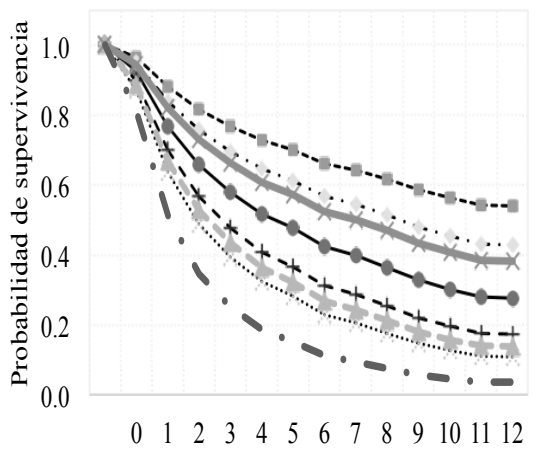

Duración en años entre el segundo y tercer hijo

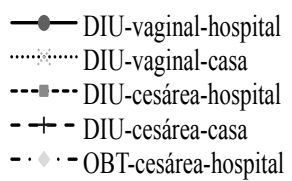

Fuente: cálculos propios con base en la EDER-2011 
Se aprecia en las tres generaciones que, una vez realizada la transición al nacimiento del tercer hijo, una proporción mayor del grupo de mujeres que no usan métodos anticonceptivos, tuvo su segundo hijo por vía vaginal y lo tuvo en casa de la mujer (No uso-vaginal-casa), tienen un tercer hijo en comparación con los otros grupos de mujeres. En todo momento tener el segundo hijo por parto vaginal es señal de una mayor probabilidad de tener un tercer hijo, independientemente del uso actual de métodos anticonceptivos o del lugar de atención del segundo hijo.

Por otro lado, los tres grupos de mujeres que tienen el mayor retraso en el calendario y una disminución de la intensidad a la transición del tercer hijo son aquellas que independientemente del uso de método anticonceptivo que usan actualmente, tuvieron a su segundo hijo por parto vía cesárea y en un hospital. La información sobre la duración mediana de los ocho grupos de mujeres en las tres cohortes de nacimiento también reafirma estas diferencias, puesto que los grupos de mujeres antes mencionados son aquellos cuya duración entre el segundo y tercer hijo es menor. Por el contrario, las duraciones más altas confirman el retraso del tercer hijo entre las mujeres que tuvieron su segundo hijo en un hospital y por cesárea.

Finalmente, se puede concluir en este apartado que tener un parto por cesárea y en un hospital durante el segundo nacimiento de un hijo son factores que disminuyen y retardan la probabilidad de tener un tercer hijo.

\section{Discusión}

La fecundidad de las mujeres indígenas residentes en el medio urbano al momento de la encuesta va en descenso, acorde a lo esperado y a lo encontrado en estudios previos a escala nacional, de forma que sus niveles de fecundidad son ligeramente superiores, pero relativamente semejantes a los del total de mujeres urbanas en las tres cohortes estudiadas en la EDER 2011.

En términos generales, al analizar los cambios en las cohortes tanto en mujeres indígenas como en las mujeres en su conjunto se observó el incremento en el nivel de acceso a la atención del parto en servicios de salud pública, acompañado del aumento en el uso de métodos anticonceptivos modernos, pero muy claramente un incremento en la intensidad y un rejuvenecimiento del calendario del uso de la OTB y no tanto del DIU, así como la proliferación del parto por cesárea, seguido de un dramático descenso del parto domiciliario en las indígenas que pasó de casi la mitad de los nacimientos tenidos por las mujeres de la cohorte avanzada a casi su desaparición en la más joven. Lo anterior no es evidencia directa de una 
práctica médica autoritaria, o que el abuso en el uso de la cesárea y de la OTB hayan sido producto de la inducción por parte del personal médico, si bien encaja en este marco explicativo. Sin embargo, consideramos que dados los casos que ya se tienen documentados en México sobre violencia obstétrica, sería menester del sector salud demostrar lo contrario.

Un análisis más fino muestra que las indígenas de la cohorte avanzada tuvieron menor acceso a los servicios públicos de atención de la salud materna y menor proporción de uso de OTB, de DIU y de cesáreas que el total de mujeres; las indígenas de la cohorte intermedia, también con menos acceso a la atención del parto en hospitales públicos, tuvieron mayor intensidad en el uso de OTB con un calendario más joven, mayor proporción de uso de DIU y de cesárea que el total de las mujeres, y; las indígenas de la cohorte joven tuvieron la misma proporción de acceso a los servicios públicos de atención a la salud materna que el total de las mujeres, mayor proporción de uso de OTB, menor de DIU y mayor proporción de partos por cesárea. Por lo tanto, las mujeres indígenas de la cohorte intermedia dieron el gran salto respecto a las de la cohorte avanzada en el incremento de la intensidad y rejuvenecimiento del uso de la OTB y del DIU, en el incremento de cesáreas y además alcanzó a superar el promedio del total de las mujeres entrevistadas en uso de OTB y de DIU con un acceso 15 puntos porcentuales menor al servicio público de atención a la salud materna.

Las trayectorias relacionadas con la vida reproductiva, sin distinción de cohortes, permiten corroborar que las más medicalizadas, como son aquellas que incluyen el parto en hospitales por cesárea o que en las que el parto fue vía vaginal, pero ocurrió en un hospital, terminan más frecuentemente con el uso del DIU o la OTB que con el no uso de métodos anticonceptivos. Aun cuando las indígenas son en mayor proporción usuarias de OTB respecto al total de mujeres, al final de las distintas trayectorias reproductivas $\mathrm{y}$ en los tres órdenes de nacimientos, los datos no indican necesariamente que las mujeres indígenas sean más vulnerables ante la medicalización a usar la OTB. Cabe ser enfáticos en que la población de estudio es urbana en el momento de la entrevista, factor que tiende a facilitar e igualar el acceso a los servicios de salud tanto para mujeres indígenas como para el total de mujeres.

Después de la llegada del tercer hijo, tal vez por tratarse del número ideal de hijos, muchas de las mujeres indígenas recurren al uso de la OTB ya sea que hubieran cursado o no una trayectoria medicalizada, a saber: la proporción de mujeres indígenas que después del tercer hijo usa OTB siendo que parió en casa por vía vaginal es de 12.8 por ciento, que es la misma 
proporción de indígenas usuarias de OTB que parieron a su tercer hijo en un hospital por vía vaginal.

Sumado a lo anterior, se examinó si las mujeres usuarias actuales de OBT y DIU, si tuvieron su hijo anterior vía cesárea y si tuvieron sus hijos en un hospital tienen una menor probabilidad de tener un segundo y tercer hijo mediante un modelo de regresión logística de tiempo discreto. El primer resultado es observar que la variable de identificación étnica no es significativa, lo cual revela que, a pesar de existir importantes diferencias entre las indígenas y el total de mujeres en el estudio de la fecundidad en México, en el medio urbano no existen en la transición al segundo y tercer hijo, y que son más explicativas otras variables tales como el tipo de parto y el lugar de atención al parto. En este sentido, los indicios confirman que más allá de ser usuaria de métodos anticonceptivos como el DIU, los otros dos factores antes mencionados son los que provocan las diferencias más significativas en cuanto al calendario y la intensidad de la transición al segundo o tercer hijo. Si bien, observamos diferencias importantes en el quantum y el tempo de los grupos de mujeres estudiados dependiendo del uso del DIU y la OBT o el no uso, estas son realmente diferentes si el parto anterior fue por cesárea en lugar de un parto vía vaginal y si el parto fue en un hospital más que en la casa de la mujer. De manera que podemos concluir diciendo que tener un parto por cesárea y en un hospital durante el primer o segundo nacimiento de un hijo son factores que disminuyen y retardan la probabilidad de tener un siguiente hijo, al menos hasta el tercero.

Respecto a la presunción de que las trayectorias asociadas con la medicalización de la fecundidad están más fuertemente relacionadas con el declive de la descendencia en las indígenas que en el total de mujeres en un contexto urbano, las cohortes intermedia y avanzada tienen más elevados niveles de uso de OTB y de cesárea que el total de mujeres urbanas, sin embargo, en términos del nacimiento del segundo y tercer hijos, el hecho de ser identificada como indígena no hace diferencias una vez controlados el tipo de parto y el lugar de atención al parto.

Finalmente, como nota crítica a los datos usados en el presente artículo, cabe mencionar que la EDER 2011 si bien tiene la ventaja de que recoge la información demográfica más relevante del encuestado desde su nacimiento hasta el momento de la entrevista; tiene como unidad temporal mínima un año calendario por lo que todo cambio ocurrido en un periodo menor de tiempo no es observable. Esta restricción deja fuera del análisis toda la variedad de anticonceptivos que se usan en el transcurso de un año y hace referencia sólo a los principales métodos empleados. Por otra parte, 
el reducido tamaño de la muestra de la EDER 2011 restringe los niveles de desagregación de las variables, lo cual es particularmente crítico en el análisis de la categoría "indígena". Esta situación se vio paliada a través de la estimación de datos suavizados con la técnica de las medias móviles y con los modelos logísticos, los cuales parten de un conjunto de datos mucho mayor al tamaño de la muestra al introducir en su análisis todos año-persona vividos por los entrevistados.

\section{REFERENCIAS BIBLIOGRÁFICAS}

Aguilar, B., 2015, "Medicalización de la vida", en Revista Uruguaya de Cardiología, vol. 30 núm. 3, pp. 262-267.

Allison, P., 2014, "Event history and survival analysis" en Quantitative Applications in the Social Sciences. Thousand Oaks, CA, SAGE Publications Inc.

Aujang, E. y Felguérez, J., 2009, "Influencia de la operación cesárea en la planificación familiar", en Revista de Ginecología Obstétrica. 77/12 2009, pp. 562-566.

Barroso, C., 2004, "La fecundidad indígena en México y Brasil. Visión oficial versus visión indígena ¿dos caminos divergentes, espejos de América Latina?”, Documento presentado en I Congrès de l'Association latino-américaine de population, ALAP, Caxambú, Brasil.

Brugeilles, C. y Rojas, O., 2016, "Inicio de la práctica anticonceptiva y formación de las familias. Experiencia de tres cohortes mexicanas", en Coubès, M.; Solís, P. y Zavala, M. (coords.), Generaciones, curso de vida y desigualdad social en México. México, El Colegio de México.

Brugeilles, C., 2014a, "Las cesáreas. ¿Un medio para regular la fecundidad?", en Coyuntura demográfica, núm. 6, pp. 35-41.

Brugeilles, C., 2014b, "L'accouchement par césarienne, un risque pour les droits reproductifs?”, en Autrepart, núm. 70, pp. 134-164.

Castro, R., 2014, "Génesis y práctica del habitus médico autoritario en México", en Revista Mexicana de Sociología, núm. 2, abril-junio, pp. 167-197.

Celi, Martha, 2018, Nivel de conocimientos y prácticas sobre la planificación familiar en las mujeres indigenas de las lagunas, Quisquinchir y Namarin del cantón Saraguro provincia de Loja, Tesis de pregrado, Universidad Nacional de Loja, Ecuador.

CONAPO, 2016, Situación de la salud sexual y reproductiva. República Mexicana, México, México, CONAPO.

Gaceta Oficial de la República Bolivariana de Venezuela, 2007, Ley orgánica sobre el derecho de las mujeres a una vida libre de violencia, Caracas.

Ham, P., 2003, "Tendencias de la mortalidad y fecundidad indígenas. Comparación con la población nacional” en González, S. (coord.), Salud y derechos repro- 
ductivos en zonas indígenas de México. Memoria del seminario de investigación. México, Documentos de trabajo núm. 12: Sexualidad, Salud y Reproducción.

IMSS, 2014, Guía de práctica clínica para la reducción de la frecuencia de operación cesárea. México, Instituto Mexicano de Seguro Social.

La Ferrara, E.; Chong, A. y Duryea, S., 2012, "Soap operas and fertility: evidence from Brazil", en American Economic Journal: Applied Economics, num. 4-4, 2012, pp. 1-31.

Lara-Contreras, Alma Concepción, Cervantes-Becerra, Roxana Gisela, Villarreal-Ríos, Enrique, Vargas-Daza, Emma Rosa, Galicia-Rodríguez, Liliana, Martínez-González, Lidia, 2018, "Prevalencia de los factores asociados a la no coincidencia del método de planificación familiar electo en la atención prenatal y el aplicado post-evento obstétrico", en Revista chilena de Obstetricia y Ginecología, 83(6), 553-559. https://dx.doi.org/10.4067/S0717-75262018000600553

Lerner, S. et al., 1994, "La pluralidad de trayectorias reproductivas y las transacciones institucionales", en Estudios Demográficos y Urbanos, vol. 9, núm. 3-27, pp.543-578.

Páez, O. y Zavala, M., 2016, “Tendencias y determinantes de la fecundidad en México: las desigualdades sociales" en Coubès, M.; Solís, P. y Zavala, M. (coords.) Generaciones, curso de vida y desigualdad social en México. México, El Colegio de México.

Palma, Y., 2005, "Políticas de población y planificación familiar", en Demos. Carta demográfica de México, núm. 16, UNAM, pp. 24-25.

Rocha-Acero, Magda Lizeth, Socarrás-Ronderos, Fernanda, Rubio-León, Diana Carolina, 2019, "Prácticas de atención del parto en una institución prestadora de servicios de salud en la ciudad de Bogotá", en Revista Facultad Nacional de Salud Pública, 37(1),53-65. Fecha de Consulta: 13/02/2020. Disponible en https://www. redalyc.org/articulo.oa?id=120/12060715010

Salgado, F. y Diaz, M., 2019, "Violencia obstétrica y de género mediante la medicalización del cuerpo femenino", en Revista Latinoamericana de Estudios sobre Cuerpos, Emociones y Sociedad, núm. 29, año 11, abril-julio. Argentina. pp. 2334.

Sartori, G., 2002, Homo videns. La sociedad teledirigida. México, Taurus.

Schooyans, M., 1997, The totalitarian trend of liberalism, St. Martin de Porres Lay Dominican Community, p. 122.

Secretaría de Salud, 2002a, Cesárea segura. Lineamiento Técnico. México, Secretaría de Salud.

Secretaría de Salud, 2002b, Anticoncepción posparto, transcesárea, poscesárea y posaborto. México, Secretaría de Salud.

Tuirán, R., 1996, “Cultura demográfica: comunicación en población y procesos de difusión”, en Demos. Carta demográfica de México, núm. 9, UNAM, pp. 25-26. 
Vázquez, G. y Cenobio, F., 2017, “Tendencias recientes de la fecundidad de las mujeres hablantes de lengua indígena en México", Ponencia del 6to. Congreso Internacional de Investigación Social, Pachuca, Universidad Autónoma del Estado de Hidalgo.

Vázquez, G. y Luna M., 2000, “Composición del desarrollo en el estado de Hidalgo. Demografía, etnicidad y pobreza”, en Ortíz, A. (coord.), México, Universidad Autónoma del Estado de Hidalgo. Pachuca, Hidalgo.

Vázquez, G., 2010, Fecundidad indigena. México, Universidad Autónoma del Estado de Hidalgo, Miguel Ángel Porrúa.

Vázquez, G., 2016, "Poblaciones indígenas urbanas en México y su comportamiento reproductivo", en Coubès, M.; Solís, P. y Zavala, M. (coords.) Generaciones, curso de vida y desigualdad social en México. México, El Colegio de México, pp.77-108.

Zavala, M., 2004, “Tendencias de la fecundidad en los tres grupos de generaciones urbanas y rurales según sexo", en Coubès, M.; Zavala, M. y Zenteno, R., (coord.), Cambios demográficos y sociales en México del siglo XX: una perspectiva de historias de vida. México, Miguel Ángel Porrúa, pp. 97-119.

\section{RESUMEN CURRICULAR DE LOS AUTORES}

Germán Vázquez Sandrin

Doctor en Estudios de las sociedades latinoamericanas con especialidad en Demografía (Université de la Sorbonne Nouvelle Paris 3, Francia). Sus principales líneas de investigación son: Demografía étnica y fecundidad. Actualmente trabaja como profesor-investigador del Área Académica de Sociología y Demografía de la Universidad Autónoma del Estado de Hidalgo.

Dirección electrónica: gevazquez@uaeh.edu.mx

Registro ORCID: https://orcid.org/0000-0002-6016-1151

\section{Elsa Ortíz Ávila}

Doctora en Demografía (Universidad Autónoma de Barcelona, España). Sus principales líneas de investigación son: Transición a la vida adulta, salud sexual y reproductiva y nupcialidad. Actualmente trabaja como profesora-investigadora del Área Académica de Sociología y Demografía de la Universidad Autónoma del Estado de Hidalgo.

Dirección electrónica: elsa_ortiz@uaeh.edu.mx

Registro ORCID: https://orcid.org/0000-0002-6016-1151

Artículo recibido el 28 de agosto de 2018 y aprobado el 9 de septiembre de 2019. 